\title{
Investigation of ex vivo Skin Penetration of Coenzyme Q10 from Microemulsions and Hydrophilic Cream
}

\author{
Efrem N. Tessema ${ }^{a} \quad K^{\prime}$ Konstanze Bosse ${ }^{a} \quad$ Johannes Wohlrab ${ }^{a, b} \quad$ Yahya Mrestani $^{a}$ \\ Reinhard H.H. Neubert ${ }^{\mathrm{a}}$ \\ anstitute of Applied Dermatopharmacy, Martin Luther University Halle-Wittenberg, Halle (Saale), Germany; \\ bDepartment of Dermatology and Venereology, Medical Faculty, Martin Luther University Halle-Wittenberg, \\ Halle (Saale), Germany
}

\section{Keywords}

Coenzyme Q10 · HPLC · Skin penetration profiles in relevant skin layers $\cdot$ Nanocarriers

\begin{abstract}
Introduction: Coenzyme Q10 (CoQ10) has been widely used in topical and cosmeceutical products due to its cutaneous antioxidant and energizer effects. CoQ10 is found in a higher concentration in the epidermis compared to dermis. The epidermal level of $\mathrm{CoQ} 10$ can be reduced due to several factors such as skin UV irradiation and photoaging. Various dermal nano-formulations have been investigated to overcome the skin barrier and enhance the poor penetration of CoQ10. The nanocarriers are designed to target and concentrate the CoQ10 in the viable epidermis. Most of these studies, however, failed to show the depth and extent of penetration of CoQ10 from the various carrier systems. Objective: The distribution of CoQ10 across the various skin layers has to be shown using skin slices representing the different skin layers. Methods: To realize this objective, a sensitive and selective HPLC method was developed and validated for the quantification of CoQ10 in the different skin slices. The method applicability to skin penetration (using excised human skin) as
\end{abstract}

karger@karger.com www.karger.com/spp

Karger $\stackrel{\text { ' }}{5}$

GOPEN ACCESS
(C) 2020 The Author(s)

Published by S. Karger AG, Basel

This is an Open Access article licensed under the Creative Commons Attribution-NonCommercial-4.0 International License (CC BY-NC) (http://www.karger.com/Services/OpenAccessLicense), applicable to the online version of the article only. Usage and distribution for commercial purposes requires written permission. well as stability studies was investigated using CoQ10-loaded lecithin-based microemulsion (ME) and hydrophilic cream formulations. Results: It could be shown that the highest concentration of $\mathrm{CoQ} 10$ in the viable epidermis, the target skin layer for CoQ10, was observed after application of the CoQ10 in the hydrophilic cream. This cream contains $10 \%$ of 2-ethylhexyl laurate which works obviously as a penetration enhancer for CoQ10. In contrast, the penetration of CoQ10 was lower from the ME. Just in the deeper dermis, a certain amount of CoQ10 could be detected. Conclusions: The HPLC method quantified the trace quantities of the CoQ10 distributed across the various skin layers and, hence, can be used to investigate the skin penetration of CoQ10 from various dermal standard and nano-formulations.

(C) 2020 The Author(s)
Published by S. Karger AG, Basel

\section{Introduction}

Coenzyme Q10 (CoQ10), also known as ubiquinone, is a key component of mitochondrial respiratory chain. It is a lipophilic molecule playing a vital role in cellular energy generation via the mitochondrial respiratory chain. Its reduced form, ubiquinol, is also an endogenous cellu- 
lar antioxidant reducing oxidative damage to lipids, proteins, and DNA caused by lipid peroxidation $[1,2]$. Due to these 2 functions, CoQ10 has been widely used in food supplements and cosmeceuticals [3].

CoQ10, as a cutaneous antioxidant and energizer, could be found in 10-fold higher levels in the epidermis than in the dermis. As the epidermis is directly exposed to environmental factors such as UV irradiation, it is also susceptible to depletion of skin antioxidants such as CoQ10 [4]. The biosynthesis of CoQ10 is reduced upon aging resulting in the reduction of its antioxidant efficiency [3]. Exogenous supplementation of CoQ10 into the skin can be beneficial in reducing photoaging. Topical administration of CoQ10-based products has been shown to prevent photoaging by reducing the level of oxidation and wrinkle depth [5]. Therefore, CoQ10 has been used as an active ingredient in topical products and cosmetics. For the cutaneous antioxidant and energizer effects, however, the CoQ10 needs to overcome the skin stratum corneum (SC) barrier and penetrate into the viable epidermis.

The high lipophilicity of CoQ10, however, resulted in poor penetration into and across the skin. The skin penetration of CoQ10 from conventional formulations is poor and cannot deliver the CoQ10 to the target skin layers. Besides, the formulations do not prolong the release of CoQ10 into the skin. Previously formulation strategies, mainly nano-sized carriers, have been designed to improve the photostability of CoQ10, promote SC penetration, to target the upper layer of skin, and achieve extended-release profile [6]. Some of the nanosized carriers investigated for dermal delivery of CoQ10 include nanostructured lipid carriers (NLCs)/ultrasmall NLCs [6-13], solid lipid nanoparticles [4, 14, 15], liposomes $[14,16]$, nano-emulsions [9], and nano-capsules $[17,18]$.

In most of the studies, the depth and extent of penetration of CoQ10 from the various carrier systems have not been shown. As formulations targeting and concentrating CoQ10 in the viable epidermis are promoted, the distribution of CoQ10 across the various skin layers has to be investigated. Therefore, the skin penetration profile of CoQ10 from various nano-sized carriers should be studied using skin slices representing the different skin layers. This requires a sensitive and selective analytical method for the detection and quantification of CoQ10 in the different skin slices and acceptor fluid in Franz diffusion cells.

Therefore, in this study, an HPLC method was developed and validated for the quantification of minute quan- tities of CoQ10 in different small-sized skin slices obtained from Franz diffusion cells. The method was applied to investigate the ex vivo skin penetration of CoQ10 from 2 formulations, microemulsion (ME) and hydrophilic cream. Furthermore, since CoQ10 is a chemically unstable compound, its short-term stability in the formulations was additionally studied.

\section{Materials and Method}

\section{Materials}

Miglyol $^{\circledR} 812$ was purchased from Caesar \& Loretz $\mathrm{GmbH}$ (Hilden, Germany). Phosal ${ }^{\circledR} 75$ SA (Phosal) was kindly donated by Phospholipid GmbH (Ludwigshafen, Germany). 1,2-pentanediol was obtained from Sigma-Aldrich Chemie GmbH (Steinheim, Germany). HPLC grade methanol and acetonitrile were purchased from VWR International S.A.S (Fontenay-sous-Bois, France).

\section{Methods}

Method Development

The HPLC system Agilent 1,220 (Agilent Technologies Deutschland GmbH, Waldbronn, Germany) was used for determination of CoQ10 in various formulations and skin samples. The CoQ10 was separated on a Eurospher II 100-5 C8 Column $(150 \times$ 20 mm KNAUER Wissenschaftliche Geräte GmbH, Berlin, Germany) under the following chromatographic conditions: methanol as mobile phase, flow rate was set at $1.2 \mathrm{~mL} / \mathrm{min}$, column temperature was $30^{\circ} \mathrm{C}$, injection volume was $30 \mu \mathrm{L}$, and total run duration was $10 \mathrm{~min}$. The CoQ10 was detected using a UV detector at $275 \mathrm{~nm}$.

Method ValidationCalibration Curve and Linearity

Calibration curves were constructed using different concentrations of CoQ10 $(0.06-10.80 \mu \mathrm{g} / \mathrm{mL})$ in acetonitrile. The linearity range of peak areas versus concentration $(\mu \mathrm{g} / \mathrm{mL})$ of CoQ10 was tested using linear regression analysis, and calibration curve parameters (correlation coefficient, slope, and intercept) were calculated.

Limits of Detection and Quantification

The limits of detection (LOD) and quantification (LOQ) of the method were estimated using signal-to-noise $(\mathrm{S} / \mathrm{N})$ ratio. Progressive dilutions of CoQ10 were prepared, and the $\mathrm{S} / \mathrm{N}$ ratios were determined using the lower 3 concentrations of calibration standards. The LOD and LOQ were considered to be the CoQ10 concentrations that produced $\mathrm{S} / \mathrm{N}$ ratio of 3 and 10 , respectively.

\section{Precision and Accuracy}

The within-run precision and accuracy of the method were determined by analyzing 4 concentration levels covering the calibration curve range (at the LLOQ, 3 times the LLOQ [QC1], medium [QC2], and at 75\% of the upper calibration curve range [QC3]) 9 times. For the validation of between-run precision and accuracy, 3 samples were analyzed on the first 2 days followed by 9 samples on the third day for each concentration. The interday and intraday variabilities of the method were expressed as coefficient of variance and percentage of the nominal value (percent recovery). 
Table 1. Compositions of LBMEs for stability and penetration studies

\begin{tabular}{lllll}
\hline MEs & $\begin{array}{l}\text { Miglyol }{ }^{\circledR} 812 \\
(\%, w t / w t)\end{array}$ & $\begin{array}{l}\text { Phosal }^{\circledR} 75 \mathrm{SA} \\
(\%, w t / w t)\end{array}$ & $\begin{array}{l}\text { Water-PD* } \\
(1.5: 8.5)(\%, w t / w t)\end{array}$ & $\begin{array}{l}\text { Q10 } \\
(\%, w t / w t)\end{array}$ \\
\hline LBME1 & 5 & 35 & 60 & 0.2 \\
LBME2 & 10 & 40 & 50 & 0.2 \\
LBME3 & 15 & 45 & 40 & 0.2 \\
\hline
\end{tabular}

ME, microemulsion; LBME, lecithin-based ME. * PD, 1,2 pentylene glycol.

\section{Formulation of CoQ10 ME and Hydrophilic Cream}

Lecithin-based MEs (LBMEs) containing CoQ10 were prepared by mixing all the components of the MEs (oil phase: Miglyol $^{\circledR} 812$, surfactant: Phosal ${ }^{\circledR} 75$ SA, and hydrophilic phase: water/1,2-pentanediol mixture [1.5:8.5]) in vials and sonicating the mixtures for $30 \mathrm{~min}$ at $40^{\circ} \mathrm{C}$ (Table 1 ).

CoQ10 (0.2\%) was incorporated into a nonionic hydrophilic cream SR (Deutscher Arzneimittel Codex, Caesar \& Loretz GmbH, Hilden, Germany). This cream is composed of nonionic emulsifying alcohols (21\%), 2-ethylhexyl laurate (10\%), glycerol (4.25\%), water $(64.04 \%)$, potassium sorbate $(0.14 \%)$, and citric acid, anhydrous $(0.07 \%)$.

\section{Stability Studies}

The chemical stability of CoQ10-based formulations was investigated for 2 weeks at refrigerator temperature, room temperature, and $40^{\circ} \mathrm{C}$. Samples were taken from each formulation on day 0,7 , and 14 . Acetonitrile $(1.8 \mathrm{~mL})$ was added to the formulations (ca. $18 \mathrm{mg}$ ) in an Eppendorf tube and vortexed (5 s), sonicated (10 $\mathrm{min})$, and centrifuged (10 $\mathrm{min}$ at 13,000 rpm). The supernatant was taken, filtered through $0.45 \mu \mathrm{m}$ PTFE syringe filter, and stored at $-20^{\circ} \mathrm{C}$ until analysis. The samples were diluted and analyzed by HPLC.

\section{Ex vivo Skin Permeation Studies}

The skin permeation of CoQ10 from the formulations was investigated using excised human skin in which the subcutaneous tissue was mechanically dissected and discarded. The skin used for permeation studies was received from the Department of Experimental Dermatology (Labor für Experimentelle Dermatologie, Universitätsklinik und Poliklinik für Dermatologie und Venerologie, Medizische Falkultät der Martin Luther University, Halle, Germany) and originated from human female (96 years) cadaver skin - breast skin. All skin donors signed an informed consent (provision of body donation for scientific purposes) available at the Institute of Anatomy and Cell Biology, Halle, Germany. After skin donation, a histological specimen was prepared and the epidermis thickness measured. Skin donors were anonymized due to privacy protection and thus given with an internal code. An assignment of the internal code is kept at the test facility, Labor für Experimentelle Dermatologie.

A circular piece of skin $\left(2 \mathrm{~cm}\right.$ in diameter, $\left.3.14 \mathrm{~cm}^{2}\right)$ was punched and mounted on a Franz diffusion cell (Crown Glass Co., Somerville, NJ, USA) with the SC side facing the donor compartment. The dermal side of the skin and the acceptor compartment were separated by filter gauze (Sartolon polyamide, pore size 0.45 $\mu \mathrm{m}$; Sartorius Stedim Biotech GmbH, Göttingen, Germany). The acceptor compartment was filled with $20 \mathrm{~mL}$ of distilled water, and

Investigation of ex vivo Skin Penetration of Coenzyme Q10
Table 2. Regression parameters of calibration curve of the HPLC method

\begin{tabular}{lccl}
\hline Parameter & Intercept & Slope & $R$ \\
\hline Day 1 & 0.062 & 22.703 & 0.9991 \\
Day 2 & 0.080 & 23.930 & 0.9999 \\
Day 3 & 0.017 & 23.250 & 0.9988 \\
Mean & 0.053 & 23.294 & 0.9993 \\
SD & 0.033 & 0.615 & 0.0006 \\
CV, \% & 61.8 & 2.6 & 0.06 \\
\hline
\end{tabular}

$\mathrm{SD}$, standard deviation.

the hydrodynamics was maintained by continuous stirring. The cell was kept at $32^{\circ} \mathrm{C}$ using circulating water. A defined amount of each formulation (ca. $20 \mathrm{mg}$ ) was applied evenly on the outer surface of the skin. The permeation experiments were carried out for $0.5,2$, and $6 \mathrm{~h}$. Following the incubation periods, the formulation remaining on the surface of the skin was carefully removed by a cotton swab and three $6-\mathrm{mm}$ diameter discs $\left(0.2827 \mathrm{~cm}^{2}\right)$ were cut out using a Kromayer punch (Stiefel-Laboratorium, Offenbach, Germany). The discs were sectioned into different slices using a cryo-microtome (Jung, Heidelberg, Germany). While the upper 10 $\mu \mathrm{m}$ thick slice represents the SC, the next four 20 - $\mu \mathrm{m}$ thick slices were considered as viable epidermal layer. Each of the dermis sublayers (DR1 to 5) was represented by five $40-\mu \mathrm{m}$ thick slices. CoQ10 in each skin layer and the remaining piece of tissue of the skin was extracted with $0.1 \mathrm{~mL}$ of acetonitrile by shaking for $1 \mathrm{~h}$, centrifuged for $10 \mathrm{~min}$ at $13,000 \mathrm{rpm}$; the supernatant was removed and kept at $-20^{\circ} \mathrm{C}$ until analysis. The amount of CoQ 10 was quantified by HPLC. The cotton swab and the gauze were extracted with 5 and $2 \mathrm{~mL}$ of acetonitrile, respectively, and followed the same treatment as the skin slices. A portion of the liquid collected from the acceptor compartment $(0.5 \mathrm{~mL})$ was dried under nitrogen stream and redissolved in $0.25 \mathrm{~mL}$ of acetonitrile and analyzed by HPLC.

\section{Results and Discussion}

\section{HPLC Method Validation}

In order to demonstrate the reliability of the HPLCUV method for the quantification of CoQ10 in skin per- 
Table 3. Three days precision and accuracy of the HPLC method for quantification of CoQ10

\begin{tabular}{|c|c|c|c|c|c|c|c|}
\hline \multirow[t]{2}{*}{ Standard } & \multirow{2}{*}{$\begin{array}{l}C_{\mathrm{nom}} \\
\mu \mathrm{g} / \mathrm{mL}\end{array}$} & \multicolumn{3}{|c|}{ Interday variability, $n=15$} & \multicolumn{3}{|c|}{ Intraday variability, $n=9$} \\
\hline & & mean $\pm \mathrm{SD}, \mu \mathrm{g} / \mathrm{mL}$ & $\mathrm{CV}, \%$ & RE, \% & mean $\pm \mathrm{SD}, \mu \mathrm{g} / \mathrm{mL}$ & $\mathrm{CV}, \%$ & RE, \% \\
\hline LLOQ & 0.0618 & $0.062 \pm 0.002$ & 2.5 & -0.1 & $0.061 \pm 0.002$ & 2.8 & -1.2 \\
\hline QC1 & 0.1854 & $0.187 \pm 0.004$ & 1.9 & 1.1 & $0.187 \pm 0.003$ & 1.7 & 0.6 \\
\hline QC2 & 4.12 & $4.223 \pm 0.069$ & 1.6 & 2.5 & $4.224 \pm 0.016$ & 0.4 & 2.5 \\
\hline QC3 & 8.24 & $8.532 \pm 0.149$ & 1.8 & 3.5 & $8.643 \pm 0.042$ & 0.5 & 4.9 \\
\hline
\end{tabular}

QC1, 3 times the LLOQ; QC2 medium; QC3, 75\% of the upper calibration curve range; SD, standard deviation; COQ10, coenzyme Q10.

Table 4. Chemical stability of CoQ10 in LBMEs and hydrophilic cream formulations

\begin{tabular}{llllll}
\hline \multirow{2}{*}{ Temperature } & Time, days & \multicolumn{2}{l}{ CoQ10 content (SD) } \\
\cline { 3 - 6 } & & LBME1 & LBME2 & LBME3 & Cream \\
\hline Refrigerator & 0 & $100.0(9.9)$ & $100.0(1.9)$ & $100.0(11.4)$ & $100.0(5.6)$ \\
& 7 & $81.7(17.3)$ & $107.5(11.7)$ & $79.1(7.1)$ & $118.0(4.7)$ \\
& 14 & $114.9(5.6)$ & $116.9(3.3)$ & $104.8(4.9)$ & $102.9(5.6)$ \\
\hline Room temperature & 0 & $100.0(9.9)$ & $100.0(1.9)$ & $100.0(11.4)$ & $100.0(5.6)$ \\
& 7 & $99.9(2.2)$ & $80.3(1.0)$ & $97.5(11.0)$ & $123.5(14.5)$ \\
& 14 & $99.9(7.1)$ & $88.8(2.8)$ & $72.0(5.3)$ & $115.8(22.7)$ \\
\hline $40^{\circ} \mathrm{C}$ & 0 & $100.0(9.9)$ & $100.0(1.9)$ & $100.0(11.4)$ & $100.0(5.6)$ \\
& 7 & $95.7(3.2)$ & $97.9(5.4)$ & $87.3(2.4)$ & $122.0(12.8)$ \\
& 14 & $115.4(8.9)$ & $106.8(3.9)$ & $95.1(5.6)$ & $110.2(1.2)$ \\
\hline
\end{tabular}

LBME, lecithin-based ME; SD, standard deviation; COQ10, coenzyme Q10.

meation studies, the method was validated in terms of sensitivity, linearity, precision, and accuracy.

\section{Linearity and Sensitivity}

The method exhibited linear responses over a concentration range of $0.06-10.80 \mu \mathrm{g} / \mathrm{mL}$ (Table 2). The LOD and LOQ of the method were estimated to be 0.012 and $0.036 \mu \mathrm{g} / \mathrm{mL}$, respectively. The method is, hence, sensitive enough to quantify the amount of CoQ10 in the formulations and skin slices.

\section{Precision and Accuracy}

The results of the method's precision and accuracy are presented in Table 3 . The coefficient of variance and percent recovery of within-run and between-run precision are within the acceptable range suggesting that the method is accurate and precise for the quantification of CoQ10 in the skin layers.

\section{Formulation Stability}

The 2 weeks stability of CoQ10 in the various ME and hydrophilic cream formulations is shown in Table 4 . CoQ10 was chemically stable in all of the studied formulations except for the LBME2 and 3 at room temperature. Therefore, the permeation studies were carried out using LBME1.

\section{CoQ10 Permeation Studies}

During ex vivo skin permeation studies, small size skin slices containing minute quantities of CoQ10 are obtained from Franz diffusion cells. The detection and quantification of CoQ10 require sensitive analytical methods. Two formulations were prepared to show the depth and extent of CoQ10 penetration as well as to check the sensitivity of the HPLC method for the quantification of CoQ10 in the various skin slices representing the different layers of the skin. The permeation profile of CoQ10 


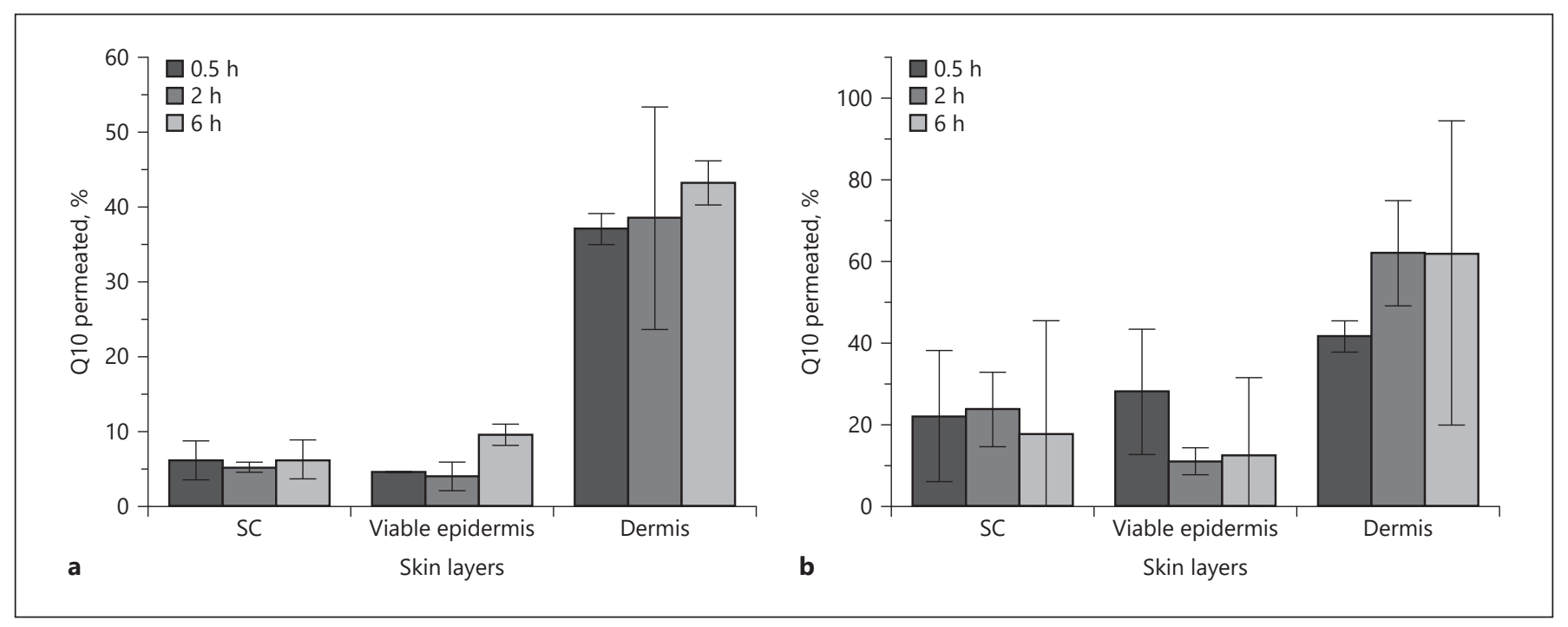

Fig. 1. Permeation of CoQ10 (\%) from LBME (a) and hydrophilic cream (b): SC: $10 \mu \mathrm{m}$ thick slice; viable epidermis: $4 \times 20 \mu \mathrm{m}$ thick slices; and dermis: $15 \times 40 \mu \mathrm{m}$ thick slices + remaining skin tissue. CoQ10 was not detected in the acceptor fluid. LBME, lecithin-based ME; SC, stratum corneum; COQ10, coenzyme Q10.

into ex vivo human skin from ME and hydrophilic cream formulations is depicted in Figure 1a and $b$.

In Figure 1 the percentage of the dose was presented on the $y$-axis to show the extent of Q10 penetrating into the skin. This is an indication for effectivity of the formulation concerning the penetration/permeation. In Figure 2 , we have used the concentration $\left(\mathrm{ng} / \mathrm{mm}^{3}\right)$ to get a real picture concerning the concentration of Q10 in the different layers of the skin because these layers have very different volumes.

It could be shown that more than $90 \%$ of the applied CoQ10 penetrated after $6 \mathrm{~h}$ into the skin from the hydrophilic cream used. In contrast, just about $60 \%$ of CoQ10 penetrated from the ME into the skin (see Fig. 1). The HPLC method enabled us to determine the quantities of CoQ10 in the various skin slices and, hence, can be used to investigate the skin layer distribution of CoQ10 from various dermal and transdermal formulations.

As shown in Figure 1, no CoQ10 was detected in the acceptor fluid of Franz diffusion cell after application of both ME and the hydrophilic cream. Surprisingly, it was observed that much higher concentrations of CoQ10 penetrated into all layers of the skin after application of the hydrophilic standard cream than after application of CoQ10 in the ME. The reason for the results appears to be that the content of lipophilic components (see Table 1, LBME1: Miglyol ${ }^{\circledR} 8125 \%$, and Phosal ${ }^{\circledR} 75$ SA 35\%) in the ME used in this study is too high, and therefore, the affin- ity of the highly lipophilic Q10 to the ME was too high. However, we had to use this content of lipophilic components in the ME because of the thermodynamic stability of the ME used. Furthermore, it could be observed in Figure 1 that the concentration of CoQ10 is decreased in the SC (after $6 \mathrm{~h}$ ) and in the epidermis (after 2 and $6 \mathrm{~h}$ ) due to penetration into the deeper layers of the skin. In contrast, the concentration of CoQ10 is decreased too in the dermis (after $6 \mathrm{~h}$ ), however, probably due to the metabolism of CoQ10 in this skin layer.

As shown in Figure 2, the highest concentration of CoQ10 in the viable epidermis, the target skin layer for CoQ10, was observed after application of the CoQ10 in the hydrophilic cream. This cream contains $10 \%$ of 2 -ethylhexyl laurate which appears to work obviously as an effective penetration enhancer for CoQ10. Similar effects were observed for the cosmetic active dihydroavenanthramide (D). The use of a mixture of propylene, butylene, and pentylene glycol in a hydrophilic cream caused the targeted accumulation of $\mathrm{D}$ in the viable epidermis and dermis of the skin [19]. In contrast, the permeation of CoQ10 was lower from the ME. Just in the deeper dermis, a certain amount of CoQ10 could be detected. Similar results were described for D (see [19]). The high concentration of the pentylene glycol (40\%) in the ME (LBME1) appears to be the reason for that result because CoQ10 has a very high solubility in pentylene glycol. This appears to be the reason that CoQ10 remains to a high percentage 


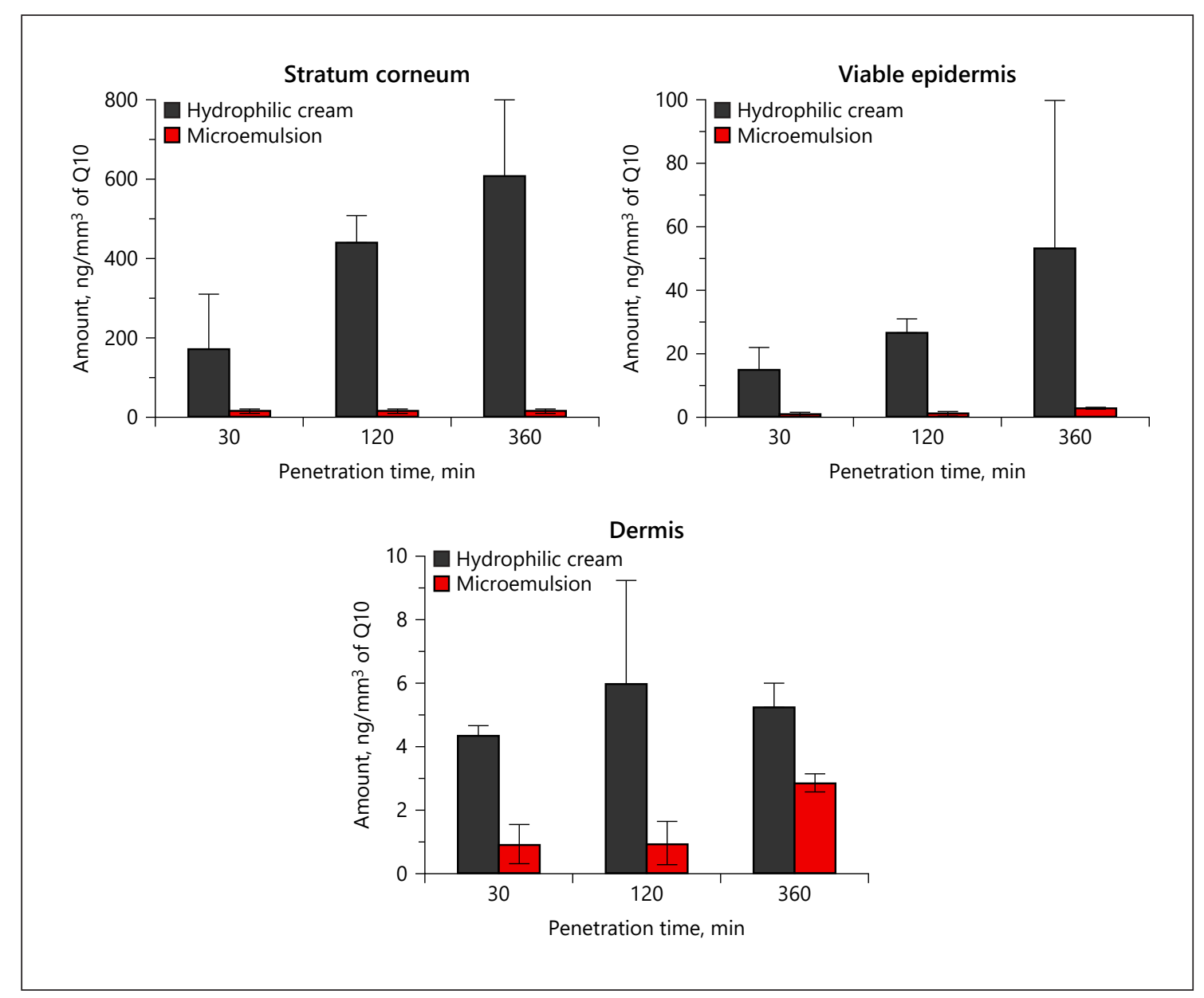

Fig. 2. Permeation of CoQ10 $\left(\mathrm{ng} / \mathrm{mm}^{3}\right)$ from hydrophilic cream and LBME: SC: $10 \mu \mathrm{m}$ thick slice; viable epidermis: $4 \times 20 \mu \mathrm{m}$ thick slices; and dermis: $25 \times 40 \mu \mathrm{m}$ thick slices + remaining skin tissue. CoQ10 was not detected in the acceptor fluid. ME, microemulsion; LBME, lecithin-based ME; SC, stratum corneum; COQ10, coenzyme Q10.

(40\%) in the ME after $6 \mathrm{~h}$. The current study demonstrated the permeation of CoQ10 into skin using ME, and hence, this formulation should be investigated in the future as a potential nanocarrier for CoQ10 to overcome the SC barrier and deliver in the viable epidermis and dermis.

\section{Conclusion}

HPLC-UV method for the detection and quantification of CoQ10 in various dermal formulations as well as skin layers was developed and validated. The method is simple and shown to be sensitive, precise, and accurate for the intended purpose. The applicability of the method to real samples (for stability as well as permeation studies) was tested using ME and hydrophilic cream containing
CoQ10. The skin permeation studies showed the amounts of the CoQ10 distributed across the various skin layers.

The highest concentration of CoQ10 in the viable epidermis, the target skin layer for CoQ10, was observed after application of the CoQ10 in the hydrophilic cream. This cream contains $10 \%$ of 2-ethylhexyl laurate which works obviously as a penetration enhancer for CoQ10.

In contrast, the permeation of CoQ10 was lower from the ME. Just in the deeper dermis, a certain amount of CoQ10 could be detected.

\section{Acknowledgements}

The authors would like to thank Claudia Bruhne and Anke Nies for their excellent technical assistance. 


\section{Statement of Ethics}

Ethical approval for permeation studies was obtained from the Department of Experimental Dermatology (Medizische Falkultät der Martin Luther University, Halle, Germany).

\section{Conflict of Interest Statement}

The authors declare no conflicts of interest.

\section{Funding Sources}

This study was supported by Institute of Applied Dermatopharmacy at the Martin Luther University Halle-Wittenberg.

\section{Author Contributions}

The contributions of the authors were equally to this paper.

\section{References}

1 Forsmark-Andrée P, Ernster L. Evidence for a protective effect of endogenous ubiquinol against oxidative damage to mitochondrial protein and DNA during lipid peroxidation. Mol Aspects Med. 1994;15 Suppl:s73-81.

2 Beyer RE. Inhibition by coenzyme Q of ethanol- and carbon tetrachloride-stimulated lipid peroxidation in vivo and catalyzed by microsomal and mitochondrial systems. Free Radic Biol Med. 1988;5(5-6):297-303.

3 Blatt T, Littarru GP. Biochemical rationale and experimental data on the antiaging properties of $\mathrm{CoQ}(10)$ at skin level. BioFactors. 2011;37(5):381-5.

4 Farboud ES, Nasrollahi SA, Tabbakhi Z. Novel formulation and evaluation of a Q10-loaded solid lipid nanoparticle cream: in vitro and in vivo studies. Int J Nanomedicine. 2011;6: 611-7.

5 Hoppe U, Bergemann J, Diembeck W, Ennen J, Gohla S, Harris I, et al. Coenzyme Q10, a cutaneous antioxidant and energizer. BioFactors. 1999;9(2-4):371-8.

6 Chen S, Liu W, Wan J, Cheng X, Gu C, Zhou $\mathrm{H}$, et al. Preparation of coenzyme Q10 nanostructured lipid carriers for epidermal targeting with high-pressure microfluidics technique. Drug Dev Ind Pharm. 2013;39(1):208.

7 Schwarz JC, Baisaeng N, Hoppel M, Löw M, Keck CM, Valenta C. Ultra-small NLC for improved dermal delivery of coenyzme Q10. Int J Pharm. 2013;447(1-2):213-7.
8 Ahmadi N, Rostamizadeh K, ModarresiAlam AR. Therapeutic anti-inflammatory potential of different formulations based on coenzyme Q10-loaded nanostructured lipid carrier: in vitro, ex vivo, and in vivo evaluations. Eur J Lipid Sci Tech. 2018;120:1800232.

9 Junyaprasert VB, Teeranachaideekul V, Souto EB, Boonme P, Müller RH. Q10-loaded NLC versus nanoemulsions: stability, rheology and in vitro skin permeation. Int J Pharm. 2009;377(1-2):207-14.

10 Lohan SB, Bauersachs S, Ahlberg S, Baisaeng N, Keck CM, Müller RH, et al. Ultra-small lipid nanoparticles promote the penetration of coenzyme Q10 in skin cells and counteract oxidative stress. Eur J Pharm Biopharm. 2015; 89:201-7.

11 Pardeike J, Schwabe K, Müller RH. Influence of nanostructured lipid carriers (NLC) on the physical properties of the cutanova nanorepair Q10 cream and the in vivo skin hydration effect. Int J Pharm. 2010;396(1-2):166-73.

12 Teeranachaideekul V, Souto EB, Junyaprasert VB, Müller RH. Cetyl palmitate-based NLC for topical delivery of Coenzyme Q(10)-development, physicochemical characterization and in vitro release studies. Eur J Pharm Biopharm. 2007;67(1):141-8.
13 Yue Y, Zhou H, Liu G, Li Y, Yan Z, Duan M The advantages of a novel CoQ10 delivery system in skin photo-protection. Int J Pharm. 2010;392(1-2):57-63.

14 Gokce EH, Korkmaz E, Tuncay-Tanriverdi S, Dellera E, Sandri G, Bonferoni MC, et al. A comparative evaluation of coenzyme Q10loaded liposomes and solid lipid nanoparticles as dermal antioxidant carriers. Int Nanomedicine. 2012;7:5109-17.

15 Korkm E, Gokce EH, Ozer O. Development and evaluation of coenzyme Q10 loaded solid lipid nanoparticle hydrogel for enhanced dermal delivery. Acta Pharm. 2013;63(4):517-29.

16 Lee WC, Tsai TH. Preparation and characterization of liposomal coenzyme Q10 for in vivo topical application. Int J Pharm. 2010;395(12):78-83.

17 Pegoraro NS, Barbieri AV, Camponogara C, Mattiazzi J, Brum ES, Marchiori MC, et al. Nanoencapsulation of coenzyme Q10 and vitamin $\mathrm{E}$ acetate protects against UVB radiation-induced skin injury in mice. Colloids Surf B Biointerfaces. 2017;150:32-40.

18 Zhou H, Yue Y, Liu G, Li Y, Zhang J, Yan Z, et al. Characterisation and skin distribution of lecithin-based coenzyme Q10-loaded lipid nanocapsules. Nanoscale Res Lett. 2010; 5(10):1561-9.

19 Heuschkel S, Wohlrab J, Neubert RH. Dermal and transdermal targeting of dihydroavenanthramide D using enhancer molecules and novel microemulsions. Eur J Pharm Biopharm. 2017;72(3):552-60. 